\title{
A Case Study: How to Restructure an Open Source Teaching Website to Improve Global and Digital Presence and Usage
}

\author{
Mike Angerbrandt ${ }^{1}$, Robert Carroll ${ }^{2}$, Adam Rosenberg $^{3}$, Marissa Swartz ${ }^{4} \&$ Maris Stella Swift ${ }^{5}$ \\ ${ }^{1}$ Chrysler Corporation, USA \\ ${ }^{2}$ Marketing, Western Michigan University, USA \\ ${ }^{3}$ Computer Science, Grand Valley State University, USA \\ ${ }^{4}$ Seidman College of Business, Grand Valley State University, USA \\ ${ }^{5}$ Management Department, Grand Valley State University, USA \\ Correspondence: Maris Stella Swift, Management Department, Grand Valley State University, USA. E-mail: \\ swiftgvsu@gmail.com
}

Received: December 5, 2014

Accepted: January 5, $2015 \quad$ Online Published: February 25, 2015

doi:10.5539/ibr.v8n3p185

URL: http://dx.doi.org/10.5539/ibr.v8n3p185

\begin{abstract}
This paper is about a team of students and faculty who receive almost a half a million dollars per year grant from Google Grants Pro to advertise their educational websites and mobile android applications. Google also provides detailed analytical tools used by the team to show the effectiveness of the websites.

In the past, the group restructured one of its websites (www.gvsu.edu/arbitration/) with its analytical data in mind to improve its effectiveness as a whole. As the change proved to be effective, the group decided to restructure a different website, Workplace Data (www.gvsu.edu/e-hr/). This paper is a summary of the changes that were made to: (1) The content of the website (2) The advertising campaign of the website and (3) How these changes improved the analytics.
\end{abstract}

Keywords: websites, higher education, teaching with technology, analytics, Google analytics, Google AdWords, developing AdWords campaigns, bounce rates, global teaching via internet, educating internet, bounce rate, Google marketing plan, click through rate, Google grant, Google impressions, YouTube for teaching, open source teaching

\section{Introduction}

\subsection{Background of Web Site}

The Workplace Data website is a teaching website focused on technology, the law, and their impact on the workplace. It is an open access website that may be used as a text or an informative source for anyone interested in the subject matter, such as professionals or students. The site hosts a collection of articles, definitions, videos and legal cases regarding technology's reach into the workplace and the law.

The website was created in 2008 under the name Electronic Human Resources or E-HR for short. Since that time, the website has undergone numerous changes. The website first served as a repository for pertinent links to news articles and case law on the web and was focused on the needs of the employer and employee in the United States. However, analytical data, captured by using Google Analytics, indicated that there was a growing user base for the site from all over the world. With this knowledge in hand, the team made a decision to redesign the website to make it easier to navigate and to focus on legal and workplace issues that impacted more than just the United States.

The changes that were made to the website and the ad campaign resulted in better analytics. The next section will explain the basics of these analytics and provide the current data so that it is possible for the reader to better understand Google Analytics.

\subsection{The Basics of Google Analytics for Our Websites}

Analytics play a major role in the development of a website. The group relies on analytics to learn information on the effectiveness of the site, such as how many people are accessing the website and how to track 
improvement in analytic metrics. Google Analytics generates statistics based on the amount of people entering the website (i.e. organic searches versus Google AdWords). This information is then transformed into metrics used to evaluate website performance. The three major analytic metrics that are being tracked in this paper are: click-through rate (CTR), bounce rate, and average time per session.

\subsubsection{CTR (Click through Rate)}

CTR is a percentage measured by taking the total number of clicks and dividing it by the total number of impressions (clicks/impressions). An impression is when an ad is viewed by a user or just placed on the page even if it is not actually seen. A click, however, is when a user views the impressions (advertisements), clicks on the ad and is then taken to the website. CTRs are very important because the group wanted to make sure they were not wasting impressions. An efficient CTR (when using Google AdWords) is considered anything over $1 \%$. For purpose of example, if an advertisement is seen one million times, a CTR would indicate that it was clicked on and viewed 10,000 times. This is a substantial number of users for educational websites.

\subsubsection{Bounce Rate}

Bounce rate is a percentage-based metric measured by distinguishing users who only visit a single page on a website. For example, if a user visited the website by clicking on an advertisement but left the website before visiting another page, then that user has bounced off the website. If a user bounces off the site quickly, it is typically a waste of money (similar to someone walking in and out of a store in seconds). However, a user could click on an advertisement which leads them exactly to needed information and then leave shortly after acquiring the legal case, video, etc. The fact of the matter is that a user not being on a page for a long time is not necessarily bad. An efficient bounce rate is anything less than $75 \%$.

\subsubsection{Average Time per Session}

Average time per session is a metric to measure the quality of a click or visit because it measures the amount of time a user spent on the site. An efficient average session time is one minute.

\subsection{Graphic Demonstration of Analytics}

The graphs below will assist the reader in understanding the analytics mentioned previously.

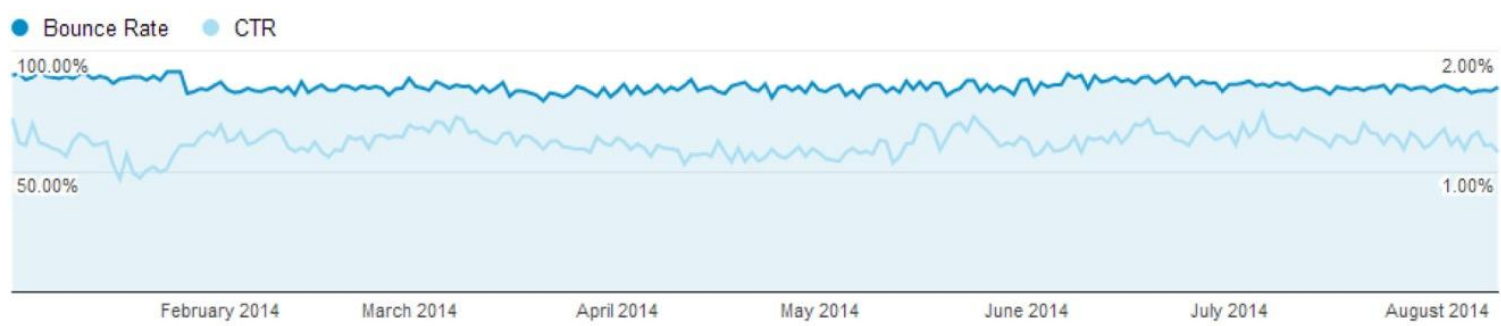

Figure 1. Bounce rate and CTR

Figure 1 shows both the bounce rate and CTR starting from the beginning of the year until August 11, 2014. The average bounce rate is $89.35 \%$ and has been as high as $95 \%$. The average CTR is $1.24 \%$ and has been as low as $.97 \%$.

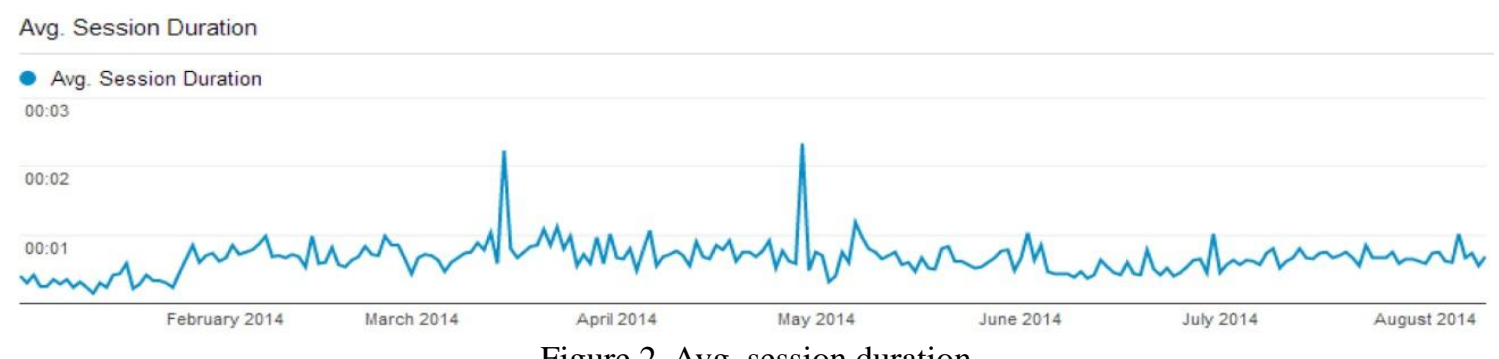

Figure 2. Avg. session duration

Figure 2 shows the average session duration from the beginning of the year until August 11, 2014. The average session time is 39 seconds and has been as low as only 9 seconds. 
The data from Figures 1 and 2 above convinced the group that there needed to be major changes to the website because the website was failing to meet basic measures of efficiency and effectiveness based on its analytical metrics. Consequently, the group decided to refocus the website's content and restructure the website's navigation. Upon the website's restructure, the group created new Google AdWords and rewrote existing advertisements. The group wanted to be sure that once an individual clicked on any AdWords the user went directly to the section of the website that was advertised.

\section{Method}

\subsection{Workplace Data Website Reinvented}

\subsubsection{Why Are Users Leaving the Website So Fast?}

As stated previously, evaluating the analytics of the Workplace Data website made it obvious to the group that changes needed to be made. With an average bounce rate of $89.35 \%$ and average session duration of 39 seconds it was clear that visitors were quickly leaving. But why? Considering the current state of the website and keeping its analytics in mind, the group found specific areas in need of change. The following issues were uncovered: (1) There was a lack of a central focus and vision, (2) The websites navigation was clumsy and difficult to use, (3) The website pages were filled with an abundance of unrelated and confusing material, and (4) There were few videos and other interactive material to engage the reader.

\subsubsection{Addressing Problems of Website}

Once such issues with the website were uncovered, it was essential to change the content of the website and restructure the Google AdWords campaign. The focus of the website became simple: teach the public the basics about how technology impacts the workplace. Navigational tools were simplified and content was made more organized and concise. Several videos were added as well as simple definitions of technology and case law.

\subsubsection{Specific Examples of Changes to Website}

Figure 3 shows the Workplace Data website before its restructure. In Figure 3, Problem One is noted. Problem One signifies a navigation panel without a clear focus and direction. Problem Two in Figure 3 represents both the lack in the overall vision of the website as well as unnecessary information related to workplace data.

Figure 4 shows the change to the first page of the website. Solution One in Figure 4 is a reinstatement of the purpose and goal of the website. The navigation panel is also more crisp and easy to understand. The removal of unrelated links was necessary to create a clear focus on the website content. The cleaner appeal of the navigation panel allows visitors to move smoothly throughout the website, decreasing the average bounce rate because visitors stay on the website longer. Solutions Two and Three offer the viewer a much cleaner look, reduced yet relevant content, and interactional videos. Three main topics are presented to visitors to click rather than eleven topics (as presented in Figure 3). The addition of interactional videos further allows visitors to explore the content of the website under a new medium. Added content with visual appeal gives visitors reason to stay on the website and explore the website's content, thus increasing the website's average session duration and further decreasing its average bounce rate.

\subsection{Graphic Demonstration of Problems and Changes to Workplace Data}

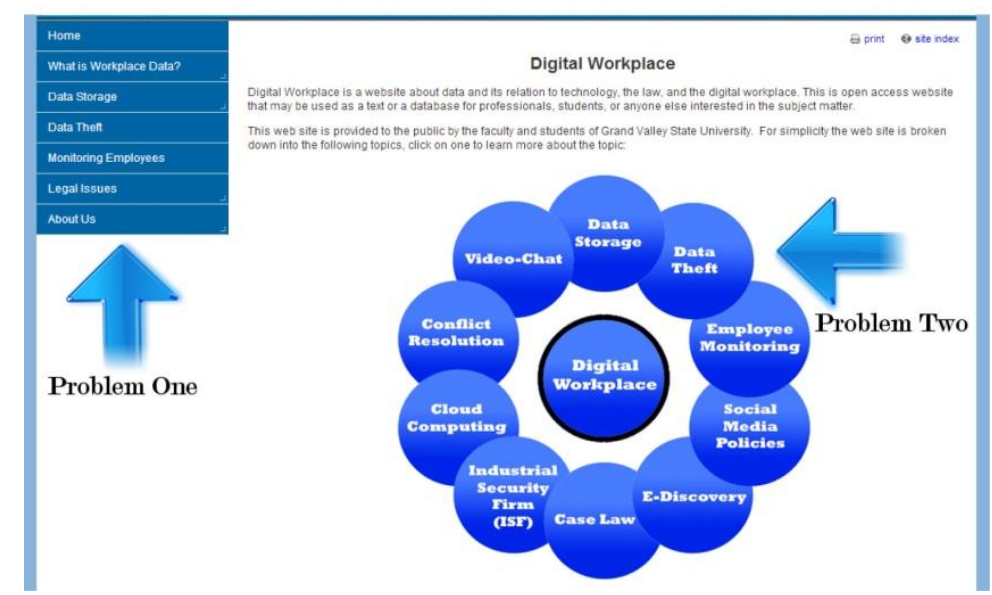

Figure 3. Main page of the workplace data website before its restructure 


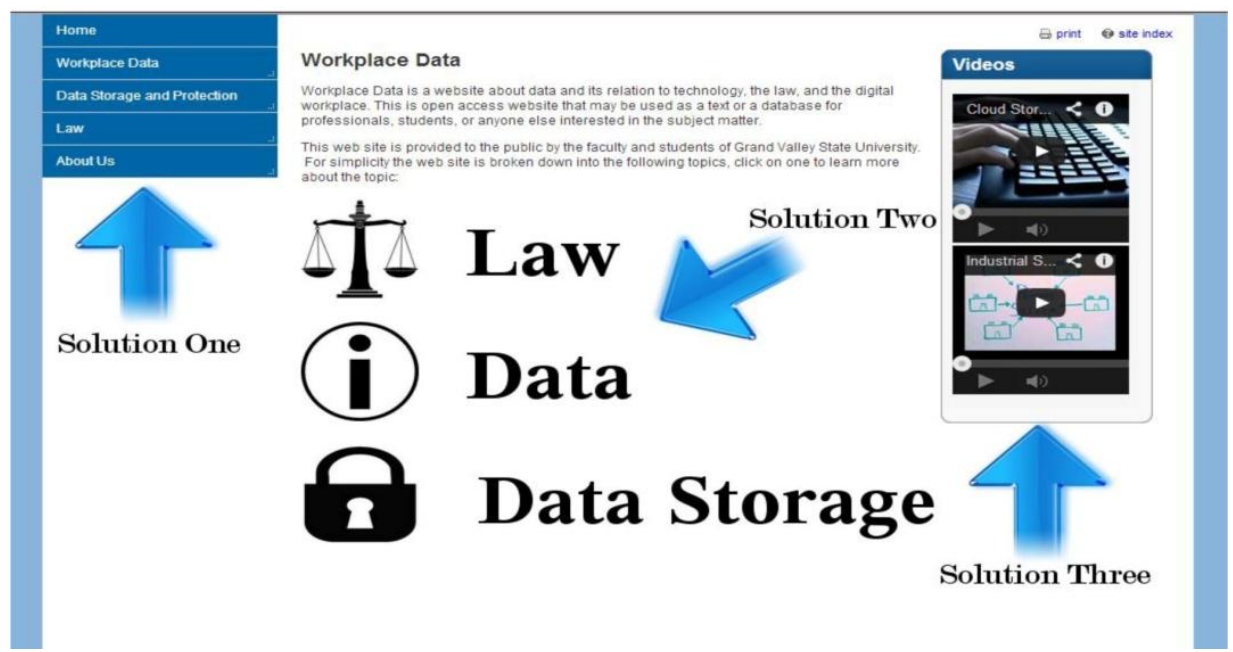

Figure 4. The workplace data website after its initial restructure

\subsection{Desired Outcomes}

The important thing to remember when working with analytics is that there is little need for guess work. The analytics indicate exactly what pages are being read, what videos are being watched, and how long users are staying on the website. The next section of the paper will explain the results and the outcome of restructuring the website and its Google AdWords.

\section{Results}

\subsection{Resulting Analytics in Teaching Website}

Table 1. Changes in analytics

\begin{tabular}{lcc}
\hline & Average Prior to Change & Current \\
\hline CTR & $1.24 \%$ & $1.35 \%$ \\
Bounce Rate & $89.35 \%$ & $85 \%$ \\
Avg. Duration & 39 seconds & 74 seconds \\
\hline
\end{tabular}

As shown in Table 1, all of the analytics show the goals for the website have been reached. First, with the website changes, the bounce rate and average duration changed drastically over a course of just one day. Second, the CTR is still well above the $1 \%$ goal. Third, the bounce rate is down to $85 \%$, and this is $5 \%$ lower than the group's goal. Fourth, the average duration on the website is 74 seconds with some pages averaging as high as 2 minutes.

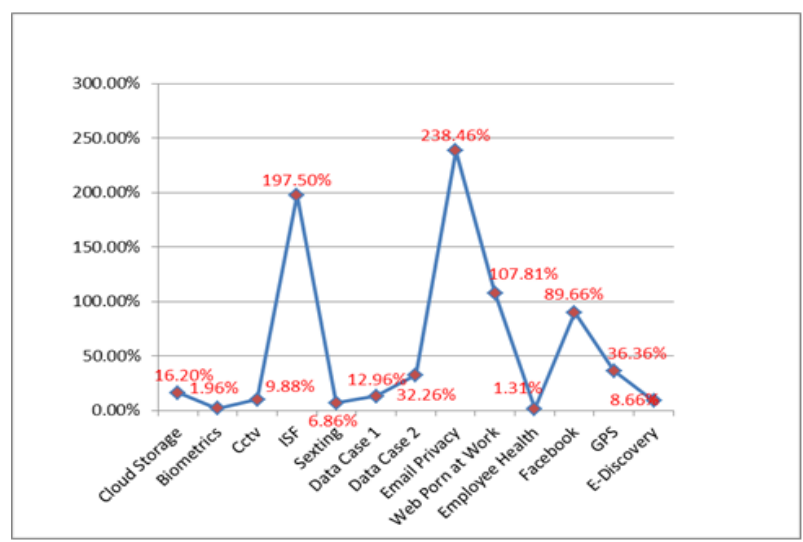

Figure 5. The increase in the percentages of video views over the last month since the website was upgraded 
Figure 5 is a graph depicting the increase in the percentages of video views over the last month since the website was upgraded. The group wanted another conversion medium to measure and decided measuring video views would be a good choice. The amount of views of some of the videos far surpassed initial goals. These videos have been online for over three years, yet in three months some of the videos tripled their view count. Many of the other videos were also viewed substantially more than in the past.

\section{Conclusion}

This paper illustrates three important facts that must be remembered in developing a website and developing a Google AdWords campaign. First, the website must have original content that is presented in a concise and easily navigable manner. Second, the Google AdWords must lead directly to the knowledge promised in the Google advertisements. Third, Google analytics allows website developers to better understand the needs of the user. These analytics are ever changing yet they are an imperative tool for any website developer.

To learn more about Team Web GVSU go to: https://www.youtube.com/watch?v=mhgJ6SuS124

\section{Acknowledgement}

We would like to acknowledge Google Grants Pro for their assistance in this research.

\section{References}

Andrykovich, A., Wisniewski, E., \& Swift, M. S. (2014). How a Google grant and Google analytics improved a Website: A case example of a University's arbitration and mediation website used by students, business and the public. International Journal of Business Administration, 5(4), 62-69. http://dx.doi.org/10.5430/ijba.v5n4p60

Bach, S., \& Haynes, P. (2006). Online learning and teaching in higher education. New York City, NY: Open University Press.

Bennett, S., Bishop, A., Dalgamo, B., Waycott, J., \& Kennedy, G. (2012). Implementing Web 2.0 technologies in higher education: A collective case study. Computers \& Education, 59(2), 524-534. http://dx.doi.org/10.1016/j.compedu.2011.12.022

Clifton, B. (2012). Advanced Web Metrics with Google Analytics. Indianapolis, IN: Sybex.

Ledford, J., \& Teixeira, J. (2010). Google analytics. Indianapolis, IN: Wiley.

Race, P. (2014). Making learning happen (3rd ed.). London, England: Sage Publications, Ltd.

Withaneachi, C., \& Johari, H. (2014). Next generation technology and the net generation: The case of a Malaysian private higher education institution. The Third International Conference on E-Learning and E-Technologies in Education (pp. 16-25).

Zikopoulos, P. (2011). Understanding big data: Analytics for enterprise class Hadoop and streaming data. New York City, NY: McGraw-Hill Osborne Media.

\section{Copyrights}

Copyright for this article is retained by the author(s), with first publication rights granted to the journal.

This is an open-access article distributed under the terms and conditions of the Creative Commons Attribution license (http://creativecommons.org/licenses/by/3.0/). 